\title{
Спин-орбитальное взаимодействие и подвижность носителей заряда в продольном автосолитоне в антимониде индия в магнитном поле
}

\author{
(С) И.К. Камилов, А.А. Степуренко , А.Э. Гумметов
}

Институт фозики Дагестанского научного центра, 367003 Махачкала, Россия

I E-mail: stepurenko-a@yandex.ru

Поступила в Редакцию 30 августа 2018 г.

В окончательной редакции 13 марта 2019 г.

Принята к публикации 27 марта 2019 г.

Предложена и рассмотрена версия о спин-орбитальном взаимодействии орбитально движущихся и спинориентированных электронов в продольном автосолитоне в антимониде индия в продольном магнитном поле. Определена дополнительная энергия, приобретаемая спин-орбитальными электронами вследствие этого взаимодействия. Получены и вычислены выражения отдельно для изменения подвижности при орбитальном движении электронов в магнитном поле и аддитивной подвижности спин-ориентированных электронов. Рассчитаны коэффициенты взаимного влияния изменяющейся подвижности электронов при орбитальном движении и аддитивной подвижности спин-ориентированных электронов.

Ключевые слова: автосолитон, электрон, спин, диамагнетизм, магнетон.

DOI: 10.21883/FTP.2019.08.47991.8978

\section{1. Введение}

Одним из примечательных явлений нелинейной физики является спонтанное или вынужденное образование пространственно неоднородных состояний - автосолитонов (AC) в неравновесной системе. В частности, такой системой может быть неравновесная и возбужденная электронно-дырочная плазма (ЭДП) в полупроводниках и полупроводниковых структурах. Создается она разными способами: фотогенерацией, ударной ионизацией, бегущими доменами Ганна, инжекцией, джоулевым разогревом и т.д. [1-9]. В зависимости от плотности плазмы в ней реализуются АС, представляющие собой микролокализованную область пониженной или повышенной концентрации носителей заряда. В электрическом поле область пониженной концентрации носителей проявляется доменом электрического поля, движущегося вдоль образца, - поперечный АC, обусловливая осцилляции тока в цепи образца. Область повышенной концентрации носителей проявляется микролокализованным шнуром тока - продольный АС. В электрическом поле в образцах $p$-InSb c концентрацией носителей $\approx 10^{12} \mathrm{~cm}^{-3}$ джоулевым разогревом генерируется ЭДП, где формируются как поперечные, так и продольные $\mathrm{AC}[7,8]$.

Единственные исследования воздействия магнитного поля на процесс формирования и эволюцию АС отражены в работах [10-17]. Поведение продольного $\mathrm{AC}$ в поперечном магнитном поле существенно отличается от поведения в продольном магнитном поле. В первом случае горячие электроны отклоняются в направлении силы Лоренца, создавая дополнительный поток тепла в этом направлении. Это приводит к раз- ности потоков на границах шнура тока. На переднем фронте постоянно происходит дополнительное прогревание материала, а на заднем - охлаждение. Таким образом, происходит движение шнура вдоль сечения образца, на краю которого или в холодной области он разрушается, приводя к повтору цикла. Это движение шнура тока представляет собой уединенную волну эффективной температуры, а вместе с ней и плотности тока [18]. Экспериментально наблюдается уменьшение тока или осцилляции тока в цепи образца [11]. Как уже было сказано $[8,9]$, продольный АC представляет собой микролокализованную область повышенной концентрации носителей заряда, то, естественно, будет происходить диффузионный отток этих носителей из центра АC на периферию. Поскольку подвижность электронов значительно превышает подвижность дырок $\left(\mu_{e} / \mu_{p} \approx b=100\right)$, вокруг малоподвижных дырок появится облако диффундирующих электронов. При включении внешнего продольного магнитного поля электроны под действием силы Лоренца начнут вращаться по орбитам вокруг малоподвижных дырок, участвуя в совместном амбиполярном дрейфе в электрическом поле $[12,13]$. Если приложить к образцу одновременно поперечное и продольное магнитные поля, то движение шнура тока в образце замедляется и при определенной величине продольного магнитного поля прекратится вовсе. Поведение АС будет уже определяться продольным магнитным полем. Вызвано это тем, что вращающиеся электроны сглаживают разность температур фронтов $\mathrm{AC}$, и волна температуры и плотности тока перестает быть таковой. Появляющийся диамагнетизм продольного АC в продольном магнитном поле [13] скажется на величине подвижности электронов ее уменьшени- 
ем $\left(\mu-\Delta \mu_{H}\right)$. Наличие продольного магнитного поля одновременно скажется на спиновой ориентации части электронов появлением некоторого количества спинориентированных вдоль этого магнитного поля электронов $\Delta n_{e}$ с аддитивной подвижностью $\Delta \mu$. Создается ситуация возможного взаимодействия суммарного орбитального магнитного момента вращающегося ансамбля электронов вокруг малоподвижных дырок со спиновым магнитным моментом спин-ориентированных электронов автосолитона, т. е. спин-орбитальное взаимодействие $(\mathrm{COB})$ в АС.

Вследствие СОВ исчезает автономность орбитального и спинового движений и возникает зацепление между ними [19]. Зацепление орбитального и спинового движений приведет, нужно полагать, к взаимному влиянию изменений параметров подвижности электронов, вызванных их орбитальным движением и спиновой поляризацией. Рассматривая электрической ток продольного АC в продольном магнитном поле противоположных направлений, можно обнаружить уменьшение токов, отличающихся по величине при смене направления магнитного поля на противоположное [15]. Проанализировав и сопоставив эти токи, можно феноменологически определить составляющие изменений подвижностей $\Delta \mu_{H}$ и $\Delta \mu$. Обусловливая диамагнетизм и парамагнетизм продольного АС в продольном магнитном поле, электроны черпают при этом энергию внешнего магнитного поля в виде некой добавки $\Delta \varepsilon$ через СОВ. Поскольку эта добавка ощутимо сказывается на поведении электронов, она стоит того, чтобы выяснить ее величину.

\section{2. Феноменологическое рассмотрение}

Рассмотрим рис. 1, где представлено графическое изображение экспериментальных осциллограмм импульсов тока АC в продольном магнитном поле противо-

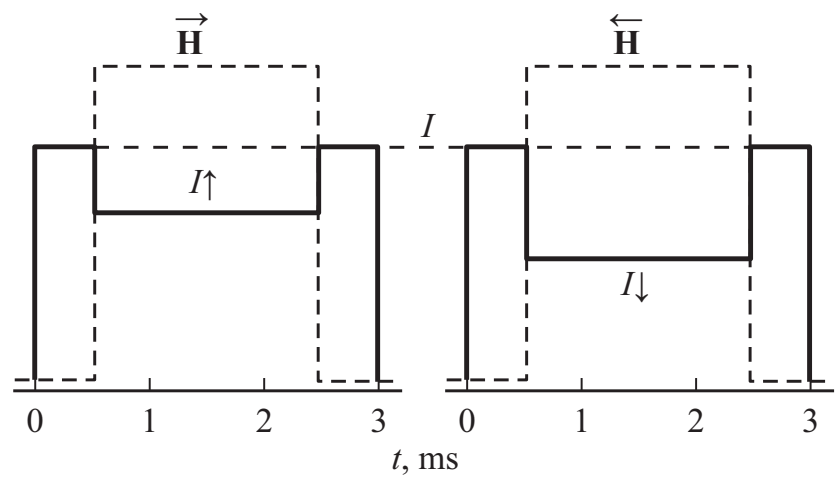

Рис. 1. Графическое изображение экспериментальных осциллограмм импульсов тока автосолитона в продольном магнитном поле противоположных направлений. $I$ - ток автосолитона, $I_{\uparrow}$ - ток автосолитона в продольном магнитном поле $\overrightarrow{\mathbf{H}}$, $I_{\downarrow}$ - ток автосолитона в продольном магнитном поле $\overleftarrow{\mathbf{H}}$. положных направлений. Оценим изменение подвижности электронов в продольном АС в продольном магнитном поле, когда электроны вращаются по некоторым орбитам и одновременно часть из них $\Delta n_{e}$ спинориентируются.

Запишем токи AC $I_{\uparrow}$ в продольном магнитном поле при $\overrightarrow{\mathbf{H}}$ и $I_{\downarrow}$ при $\overleftarrow{\mathbf{H}}$

$$
\begin{aligned}
I_{\uparrow}= & e E S\left[n_{e}\left(\mu_{a}-\Delta \mu_{H}+C \Delta n_{e} \Delta \mu / n_{e}\right)\right. \\
& \left.+\Delta n_{e}\left(\Delta \mu-P n_{e} \Delta \mu_{H} / \Delta n_{e}\right)+n_{p} \mu_{a}\right] \\
= & e E S\left[n_{e}\left(\mu_{a}-\Delta \mu_{H}+C \beta \Delta \mu\right)+n_{e} \beta \Delta \mu-P n_{e} \Delta \mu_{H}+n_{p} \mu_{a}\right] \\
= & (e n E S / 2)\left[2 \mu_{a}-(1+P) \Delta \mu_{H}+(1+C) \beta \Delta \mu\right] ; \\
I_{\downarrow}= & e E S\left[n_{e}\left(\mu_{a}-\Delta \mu_{H}-C \Delta n_{e} \Delta \mu / n_{e}\right)\right. \\
& \left.+\Delta n_{e}\left(-\Delta \mu-P n_{e} \Delta \mu_{H} / \Delta n_{e}\right)+n_{p} \mu_{a}\right] \\
= & e E S\left[n_{e}\left(\mu_{a}-\Delta \mu_{H}-C \beta \Delta \mu\right)-n_{e} \beta \Delta \mu-P n_{e} \Delta \mu_{H}+n_{p} \mu_{a}\right] \\
= & (e n E S / 2)\left[2 \mu_{a}-(1+P) \Delta \mu_{H}-(1+C) \beta \Delta \mu\right],
\end{aligned}
$$

где $E$ - напряженность электрического поля, приложенного к образцу; $n_{e}, n_{p}$ - концентрации электронов и дырок в $\mathrm{AC}, n_{e}=n_{p}=n_{\mathrm{AC}} / 2 ; S-$ площадь поперечного сечения $\mathrm{AC}, \mu_{a}$ - амбиполярная подвижность электронов и дырок, $\mu_{a}=2 \mu_{p} ; \Delta n_{e}=\left(\mu_{0} \mu_{\mathrm{B}} g^{2} / 4 \varepsilon_{\mathrm{F}}\right) H n_{e}=\beta n_{e}-$ число спин-ориентированных электронов в магнитном поле; $C-$ коэффициент, учитывающий влияние $\Delta \mu_{H}$ на $\Delta \mu$ при $\mathrm{COB} ; P$ - коэффициент, учитывающий влияние $\Delta \mu$ на $\Delta \mu_{H}$ при $\mathrm{COB} ; \mu_{0}-$ магнитная постоянная, $\mu_{\mathrm{B}}$ - магнетон Бора, $g=-56-g$-фактор электрона в $p$-InSb [20], $\varepsilon_{\mathrm{F}}$ - энергия Ферми.

Рассмотрим сумму этих токов и их разность.

$$
\begin{gathered}
I_{\uparrow}+I_{\downarrow}=(e n E S / 2)\left[4 \mu_{a}-2(1+P) \Delta \mu_{H}\right], \\
(1+P) \Delta \mu_{H}=2 \mu_{a}-\left(I_{\uparrow}+I_{\downarrow}\right) / e n E S, \\
\Delta \mu_{H}=\left\{\left[2 I-\left(I_{\uparrow}+I_{\downarrow}\right)\right] / I(1+P)\right\} \mu_{a}, \\
I_{\uparrow}-I_{\downarrow}=(e n E S / 2)[2(1+C) \beta \Delta \mu], \\
(1+C) \beta \Delta \mu=\left(I_{\uparrow}-I_{\downarrow}\right) / e n E S, \\
\Delta \mu=\left[\left(I_{\uparrow}-I_{\downarrow}\right) / I(1+C) \beta\right] \mu_{a} .
\end{gathered}
$$

Поскольку СОВ происходит из-за взаимодействия орбитального магнитного момента электронов $M_{0}$ и магнитного момента спин-ориентированных электронов $M_{p}$, естественно предположить: $C \sim M_{0}, P \sim M_{p}$, $C / P=M_{0} / M_{p}$. Если считать совместный вклад $\Delta \mu_{H}$ и $\Delta \mu$ при СОВ за единицу, можно принять $C+P=1$. Получается: $C=M_{0} /\left(M_{0}+M_{p}\right), \quad P=M_{p} /\left(M_{0}+M_{p}\right)$. Известно [17]: $M_{0}=\left(-0.2 \pi \mu_{0} e^{2} L^{4} \ln _{e} / m_{e}\right) H_{0}^{2} / H_{n}$ $=-A H_{0}^{2} / H_{n}, \quad M_{p}=\chi H_{v}=B H, \quad \chi=n_{e} \mu_{0} \mu_{\mathrm{B}}^{2} g^{2} / 4 \varepsilon_{\mathrm{F}}-$ парамагнитная восприимчивость АC $[14], L=10^{-5} \mathrm{M}-$ поперечный размер $\mathrm{AC}, l$ - длина $\mathrm{AC}$ (образца), 
$v=l \cdot S=\pi L^{2} l / 4-$ объем АС. Принимая все это во внимание, получаем

$$
\begin{gathered}
C=\left(-A H_{0}^{2} / H_{n}\right) /\left(-A H_{0}^{2} / H_{n}+B H\right) \\
=A /\left(A-B H_{n}^{2} / H_{0}^{2}\right)=A /\left(A-B n^{2}\right), \\
P=B H /\left(-A H_{0}^{2} / H_{n}+B H\right) \\
=\left(-B H_{n}^{2} / H_{0}^{2}\right) /\left(A-B H_{n}^{2} / H_{0}^{2}\right)=-B n^{2} /\left(A-B n^{2}\right),
\end{gathered}
$$

где $n=H_{n} / H_{0} \quad$ или $\quad H_{n}=n H_{0}, \quad n=1,2,3, \ldots n$, $H_{0}=2 \cdot 10^{3} \mathrm{~A} / \mathrm{M}$ - минимальное магнитное поле, при котором совершается один цикл вращения электрона за время диффузионного смещения; $H_{n}-$ магнитное поле, при котором совершается $n$ циклов вращения электрона за время диффузионного смещения, $n-$ число циклов вращения.

\section{3. Эксперимент}

Необходимые экспериментальные исследования проводились на образцах $p$-InSb c концентрацией носителей заряда $p=1.9 \cdot 10^{12} \mathrm{~cm}^{-3}$ с подвижностью $\mu_{p}=6870 \mathrm{~cm}^{2} / \mathrm{B} \cdot \mathrm{c}$ при температуре жидкого азота $T=77 \mathrm{~K}$. За температуру АC $T_{\mathrm{AC}}$ можно принять температуру, при которой появляется собственная проводимость в $p$-InSb. Экспериментально полученная температурная зависимость электропроводности образца $\sigma(T)$ дает $T_{\mathrm{AC}} \approx 150 \mathrm{~K}$. Отсюда подвижность носителей заряда в АС будет составлять $\mu_{p}(150 \mathrm{~K})=\mu_{p}(77 \mathrm{~K}) \cdot(150 / 77)^{-2.1}=1697 \mathrm{~cm}^{2} / \mathrm{B} \cdot \mathrm{c} \quad[20]$. Измерению подвергалось несколько образцов, отличающихся линейными размерами. Длина образца, вдоль которого прикладывалось электрическое поле $E=V / l$, варьировалась в пределах $0.1-0.02 \mathrm{~cm}$, поперечные размеры $a \times b=0.04 \mathrm{~cm}^{2}$. Выбор таких размеров позволял избегать появления поперечных $\mathrm{AC}$, проявляющимися осцилляциями тока в цепи образца, что давало возможность иметь дело только с продольными АС. Исследования заключались в реализации вольт-амперных характеристик (BAX) и гаусс-амперных характеристик тока АC (ГАХ) как в импульсном, так и динамическом режимах прикладываемых электрического и магнитного полей. А именно приложением импульса электрического поля длительностью $\tau_{E}$ к образцу, помещенного в соленоид, возбуждали АС с заданным значением тока $I_{\mathrm{AC}}$. Синхронно создавалось в соленоиде магнитное поле длительностью $\tau_{H}<\tau_{E}$. Фиксировалась точка $I(H)$. C помощью таких точек, полученных при нарастании магнитного поля, строилась ГАХ. В случае динамического режима во время приложенного импульса электрического поля $\tau_{E}$ в соленоиде формировался импульс магнитного поля треугольной формы длительностью $\tau_{H}<\tau_{E}$. Длительность импульса электрического поля составляла $\tau_{E}=3$ мс, а длительность импульса магнитного поля $\tau_{H}=2$ мс. Величина магнитного поля не превышала
$8 \cdot 10^{4} \mathrm{~A} / \mathrm{M}$. Величина напряжения электрического поля не превышала десятка вольт.

\section{4. Результаты и обсуждение}

Предлагаются вниманию типичные результаты экспериментальных исследований одного из нескольких образцов. На рис. 2, а представлены ВАХ образца $(l=0.05 \mathrm{~cm})$ в отсутствие $(H=0)$ и в присутствии внешнего продольного магнитного поля противоположных направлений $(|\overrightarrow{\mathbf{H}}|=|\overleftarrow{\mathbf{H}}|)$. Токи на нелинейном участке ВАX АC при воздействии магнитного поля всегда оказывались меньше. Отличались токи по величине и при переключении направления продольного магнитного поля на противоположное. На рис. 2, $b$ представлены ГАХ тока продольного АС при противоположных направлениях продольного магнитного поля. Уменьшение токов с ростом продольного магнитного поля обусловлено не иначе, как изменением подвижности носителей заряда на величину $\Delta \mu_{H}$ по причине искривления траекторий радиально движущихся электронов. Явное различие линий то-

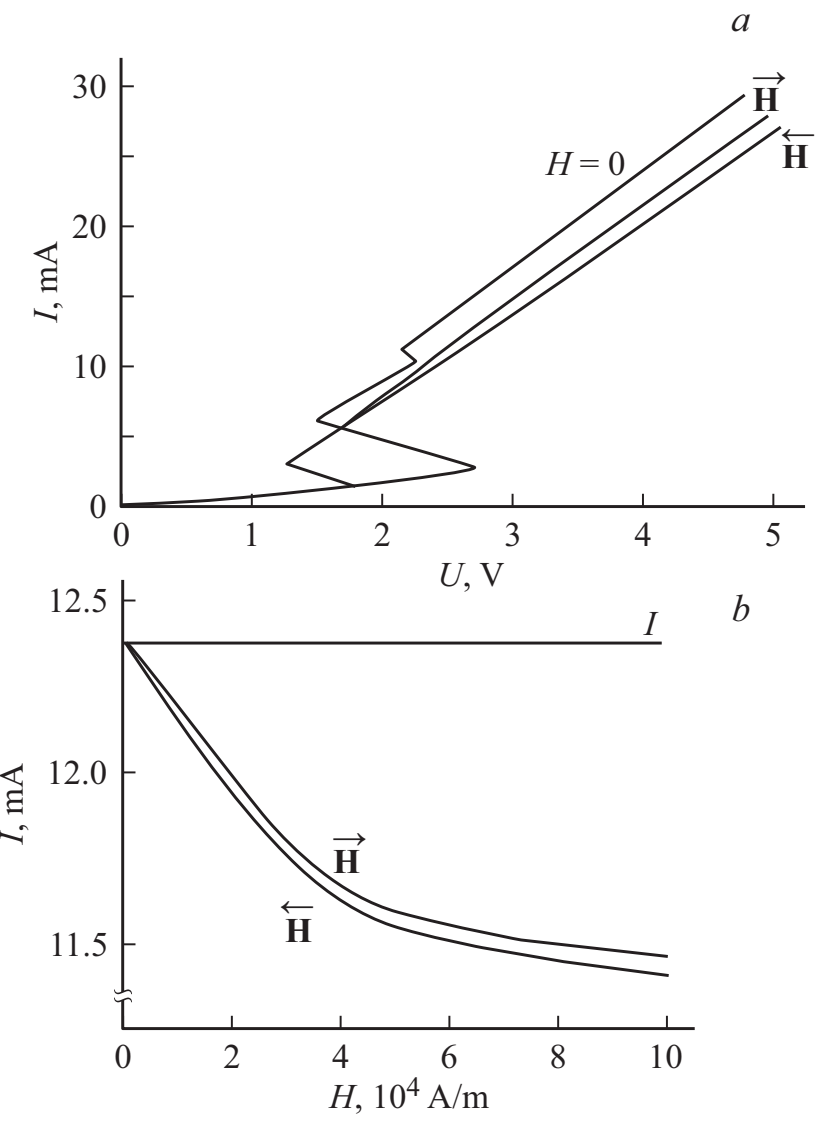

Рис. 2. $a-$ вольт-амперные характеристики продольного автосолитона в продольном магнитном поле противоположных направлений $\left(H=8 \cdot 10^{4} \mathrm{~A} / \mathrm{M}\right), b$ - гаусс-амперные характеристики тока продольного автосолитона в продольном магнитном поле противоположных направлений. 
ка на ГАХ при $|\overrightarrow{\mathbf{H}}|=|\overleftarrow{\mathbf{H}}|$ вызвано неким параметром ЭДП, конкретнее электронов, реагирующих именно на направление магнитного поля. Таким параметром является спин электрона $s_{\uparrow \downarrow}$, ориентирующийся вдоль приложенного магнитного поля. В магнитном поле, согласно предложенному Паули механизму парамагнетизма в АC, являющемся вырожденной ЭДП $\left(n=0.31 \cdot 10^{18} \mathrm{~cm}^{-3}\right)$, появится некоторый избыток электронов $\Delta n_{e \uparrow}=\Delta n_{e \downarrow}=\Delta n_{e}=n_{e} \beta$, спин которых направлен вдоль приложенного магнитного поля. Магнитный момент этих поляризованных по спину электронов, как показано [15], будет $M_{p}=\Delta n_{e} \mu_{\mathrm{B}} v=n_{e} \mu_{\mathrm{B}} \beta v$. Вращающиеся вокруг дырок электроны создают магнитное поле $H^{0}=-M_{0} / v$, которое будет воздействовать на спиновый магнитный момент поляризованных электронов $M_{p}$. Электроны получат дополнительную энергию $\Delta \varepsilon$, обусловленную этим взаимодействием и зависящую от ориентации их спинов: $\Delta \varepsilon=-H^{0}\left( \pm M_{p}\right)=\mp\left(M_{0} / v\right) \cdot n_{e} \mu_{\mathrm{B}} \beta v=\mp M_{0} n_{e} \mu_{\mathrm{B}} \beta$. Pacсматривая выражение

$$
\begin{aligned}
\Delta \varepsilon & =\left(\mp 0.2 \pi e^{2} n_{e}^{2} l L^{4} \mu_{0}^{2} \mu_{\mathrm{B}}^{2} g^{2} H_{0}^{2} / 4 m_{e} \varepsilon_{\mathrm{F}}\right) \cdot H / H_{n} \\
& =\mp \Delta \varepsilon_{0} H / H_{n},
\end{aligned}
$$

следует отметить, что при $H=H_{n}$ отношение $H / H_{n}=1$, $\Delta \varepsilon=\mp \Delta \varepsilon_{0} \cdot 1$ во всем интервале магнитного поля. Результаты имеют смысл при $H \geq H_{1} \geq H_{0} . H_{n}=n H_{0}-$ фиксированные значения магнитного поля. При $\mathrm{H}<\mathrm{H}_{2}$ $H / H_{1}<2$, при $H<H_{3} \quad H / H_{2}<3 / 2$, при $H<H_{4}$ $H / H_{3}<4 / 3$ и т.д. $H / H_{n}<n /(n-1) . \Delta \varepsilon=\mp \Delta \varepsilon_{0}$ при $H=H_{n} ; \Delta \varepsilon=\mp \Delta \varepsilon_{0} n /(n-1)$ при $H<H_{n}$. Благодаря тому, что при СОВ у электронов появляется дополнительная энергия $\Delta \varepsilon=\mp \Delta \varepsilon_{0} \cdot n /(n-1)$, электроны со спином „вверх“ и „вниз“ приобретают различный дрейфовый импульс в электрическом поле [21]. Возникает аддитивная подвижность спин-ориентированных электронов $\mu_{e \uparrow}=\mu_{e}+\Delta \mu, \mu_{e \downarrow}=\mu_{e}-\Delta \mu$. Различие подвижностей спин-ориентированных электронов „вверх“ и электронов „Вниз“, скорее всего, определяют отличие линий тока на ГАХ.

Энергия $\Delta \varepsilon$ обнаруживает затухающее биение своей величины. При известных параметрах образца, постоянных величин и выражениях для $M_{0}$ и $M_{p}$ получим в окончательном виде: $\Delta \varepsilon=\mp 23.75 n /(n-1)$ эВ. График этой зависимости представляет собой циклическое сохранение постоянной величины $\varepsilon_{0}=\mp 23.75$ эВ (с периодом $n=1,2,3, \ldots)$ с затухающими выбросами в промежутках $\left(\lim _{n \rightarrow \infty} \frac{n}{n-1}=1\right)$ (рис. 3).

Рассматриваемая версия СОВ в АС позволяет оценить изменение подвижности электронов при их вращательном движении в магнитном поле и спинориентированных электронов в этом же поле. Общее изменение подвижности получаем из результатов экспериментальных исследований поведения тока АС в продольном магнитном поле. Составляющую изменения подвижности носителей при вращательном движении

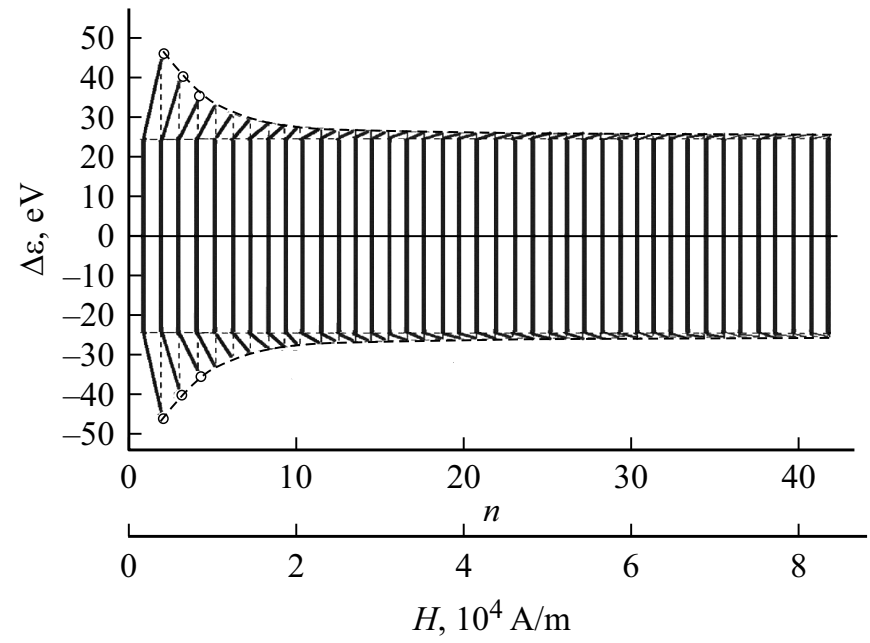

Рис. 3. Дополнительная энергия, приобретаемая спин-ориентированными электронами при СОВ в автосолитоне с ростом продольного магнитного поля $\left(H_{n}=n \cdot 2 \cdot 10^{3} \mathrm{~A} / \mathrm{M}\right)$.

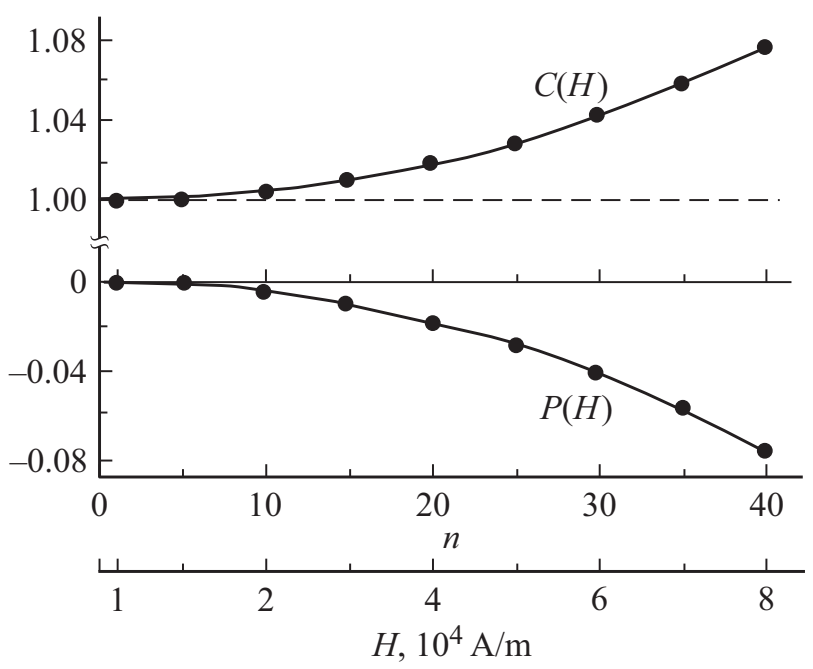

Рис. 4. Коэффициенты $C$ и $P$ взаимовлияния при СОВ изменяющейся подвижности при орбитальном движении электронов и аддитивной подвижности спин-ориентированных электронов в продольном автосолитоне в продольном магнитном поле.

в магнитном поле $\Delta \mu_{H}$ позволяет получить выражение (3). Составляющую изменения подвижности за счет спиновой ориентации части электронов $\Delta \mu$ получим из выражения (4).

Для рассматриваемого образца $M_{0}=$ $=-1.17 \cdot 10^{-12} \cdot H_{0}^{2} / H_{n} \mathrm{~A} / \mathrm{M}^{2}, M_{p}=5.11 \cdot 10^{-17} \cdot H \mathrm{~A} / \mathrm{M}^{2}$. Подставляя в выражения (5) и (6), получим: $C=1$ / $\left(1-4.37 \cdot 10^{-5} n^{2}\right), P=-4.37 \cdot 10^{-5} n^{2} /\left(1-4.37 \cdot 10^{-5} n^{2}\right)$.

На рис. 4. представлены графики зависимости $C$ и $P$ от магнитного поля. Можно заметить, что в интервале значений магнитного поля, используемого в экспериментах, величина $C$ изменяется незначительно и близка к 1 $(C \approx 1)$, а величина $P$ также изменяется незначительно 

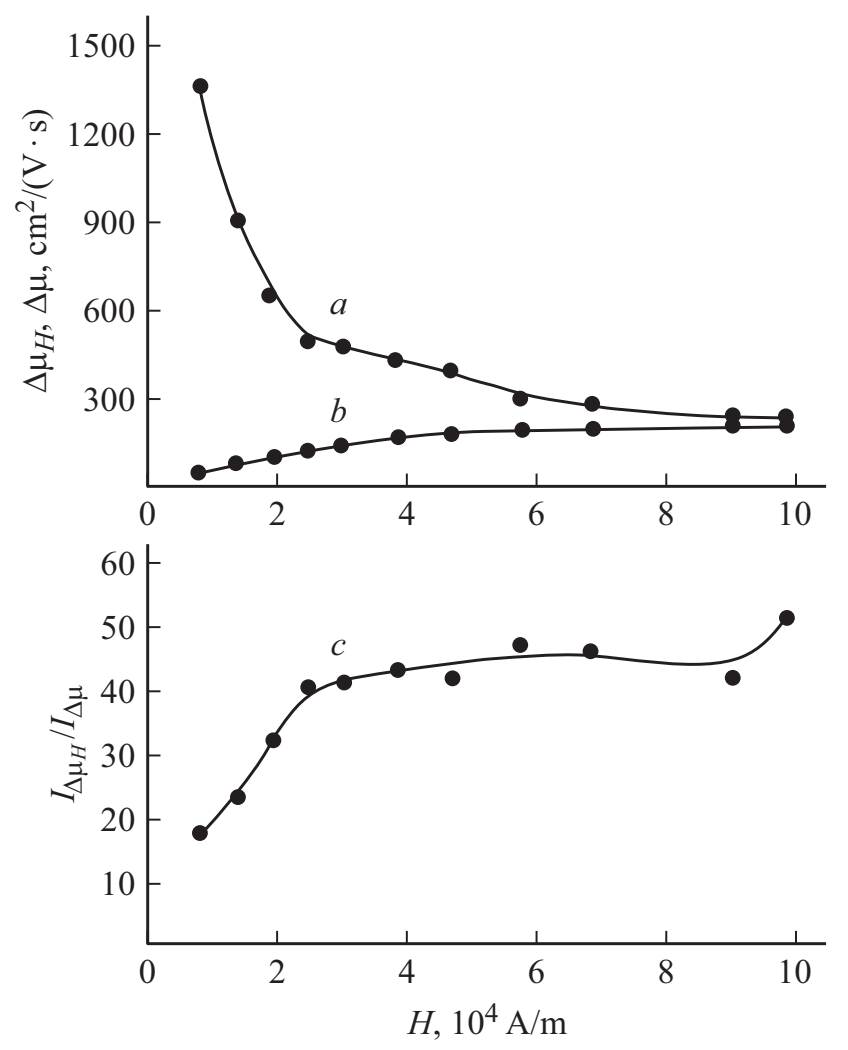

Рис. 5. $a-\Delta \mu-$ изменение аддитивной подвижности спинориентированных электронов в продольном автосолитоне в продольном магнитном поле, $b-\Delta \mu_{H}-$ изменение подвижности электронов при орбитальном движении в продольном автосолитоне в продольном магнитном поле, $c-$ соотношение токов $I_{\Delta \mu_{H}} / I_{\Delta \mu}$ в автосолитоне, появляющихся в продольном магнитном поле из-за проявления $\Delta \mu$ и $\Delta \mu_{H}$.

и близка к $0(P \approx 0)$. Выражения для $\Delta \mu_{H}$ и $\Delta \mu$ можно записать:

$$
\begin{gathered}
\Delta \mu_{H}=\left\{\left[2 I-\left(I_{\uparrow}+I_{\downarrow}\right)\right] / I\right\} \mu_{a}, \\
\Delta \mu=\left[\left(I_{\uparrow}-I_{\downarrow}\right) / 2 I \beta\right] \mu_{a} .
\end{gathered}
$$

Получается, что $\Delta \mu_{H}$ не изменяется вследствие СОВ, поскольку спиновый магнитный момент $M_{p}$ слишком мал и вкладом его в СОВ можно пренебречь в измеряемом интервале магнитного поля. Что касается $\Delta \mu$, то оно, согласно выражению (8), заметно уменышается из-за того, что орбитальный магнитный момент $M_{0}$ максимален при магнитных полях, приходящихся на начало измеряемого интервала, и вклад его в СОВ преимуществен. Графическая реализация аналитических выражений (7) и (8) с учетом экспериментальных результатов (рис. 2, $b$ ) осуществлена на рис. 5, $a, b$. Видно, что аддитивная подвижность спин-ориентированных электронов $\Delta \mu$ превышает или сравнима с изменением подвижности всех электронов $\Delta \mu_{H}$ в магнитном поле. Однако, если оценить вклад тока всех носителей с подвижностью $\Delta \mu_{H} I_{\Delta \mu_{H}}=e E S n_{e} \Delta \mu_{H}$ и вклад тока спин-ориентированных электронов с аддитивной подвижностью $\Delta \mu$
$I_{\Delta \mu}=e E S \Delta n \Delta \mu=e E S n_{e} \beta \Delta \mu$ в общий ток $I$, то получим $I_{\Delta \mu_{H}}>I_{\Delta \mu}$. На рис. 5, $c$ показано соотношение этих токов $I_{\Delta \mu_{H}} / I_{\Delta \mu}=\Delta \mu_{H} / \Delta \mu \beta=2\left[2 I-\left(I_{\uparrow}+I_{\downarrow}\right)\right] /\left(I_{\uparrow}-I_{\downarrow}\right)$.

\section{5. Заключение}

Феноменологически определены изменения подвижности $\Delta \mu_{H}(H)$ по причине искривления траектории движения электронов и аддитивная подвижность $\pm \Delta \mu(H)$ спин-ориентированных электронов. Наличие в продольном АС в продольном магнитном поле орбитально движущихся и спин-ориентированных электронов позволило воспользоваться версией о СОВ для феноменологического рассмотрения взаимовлияния возникших изменений подвижностей $\Delta \mu_{H}$ и $\Delta \mu$. Получены выражения введенных коэффициентов $C$ и $P$, учитывающих взаимовлияние подвижностей $\Delta \mu_{H}$ и $\Delta \mu$ при СОВ и их графические изображения для конкретных образцов, откуда следует, что $C \approx 1, P \approx 0$ в используемом интервале магнитного поля.

Вычислены с учетом параметров конкретных образцов известные выражения для орбитального и спинового магнитных моментов:

$$
\begin{aligned}
M_{0} & =\left(-0.2 \pi \mu_{0} e^{2} L^{4} \ln _{e} / m_{e}\right) \cdot H_{0}^{2} / H_{n} \\
& =-1.17 \cdot 10^{-12} \cdot H_{0}^{2} / H_{n} \mathrm{~A} / \mathrm{M}^{2}
\end{aligned}
$$

$M_{p}=\left(n_{e} \mu_{0} \mu_{\mathrm{B}}^{2} g^{2} v / 4 \varepsilon_{\mathrm{F}}\right) \cdot H=5.11 \cdot 10^{-17} \cdot H \mathrm{~A} / \mathrm{M}^{2} \quad$ соответственно.

Произведена оценка дополнительной энергии, которую получают спин-ориентированные электроны при COB

$$
\begin{aligned}
\Delta \varepsilon & =\left(\mp 0.2 \pi e^{2} n_{e}^{2} l L^{4} \mu_{0} \mu_{\mathrm{B}}^{2} g^{2} H_{0} / 4 m_{e} \varepsilon_{\mathrm{F}}\right) H / H_{n} \\
& =\mp 23.75\left(H / H_{n}\right) \text { эВ }=\mp 23.75 \text { эВ }
\end{aligned}
$$

при $H=H_{n}$ во всем интервале магнитного поля.

\section{Финансирование работы}

Работа выполнена в рамках выполнения государственного задания Министерства науки и высшего образования Российской Федерации (тема: AAAA-A17-1170213 10366-5).

\section{Конфликт интересов}

Авторы заявляют, что у них нет конфликта интересов.

\section{Список литературы}

[1] T. Hayashi, T. Morita, M. Fukaya, E. Hasegawa. Japan J. Appl. Phys., 13 (10), 1667 (1974).

[2] Б.С. Кернер, В.Ф. Синкевич. Письма ЖЭТФ, 36, 359 (1982).

[3] Б.С. Кернер, В.В. Осипов, М.Т. Романко, В.Ф. Синкевич. Письма ЖЭТФ, 44 (2), 77 (1986). 
[4] В.А. Ващенко, Б.С. Кернер, В.В. Осипов, В.Ф. Синкевич. ФТП, 23, 1378 (1989).

[5] М.Н. Винославский. ФТТ, 31, 315 (1989).

[6] R. Symanczyk, S. Gaelings, D. Jäger. Phys. Lett. A, 160. 397 (1991).

[7] А.А. Степуренко. ФТП, 28, 402 (1994).

[8] А.А. Степуренко. ФТП, 30, 76 (1996).

[9] И.К. Камилов, А.А. Степуренко, А.С. Ковалев. ФТП, 38 (7), 822 (2004).

[10] И.К. Камилов, А.А. Степуренко, А.С. Ковалев, А.Э. Гумметов. ФТП, 41 (3), 286 (2007).

[11] И.К. Камилов, А.А. Степуренко, А.С. Ковалев, А.Э. Гумметов. ФТП, 42 (4), 393 (2008).

[12] И.К. Камилов, А.А. Степуренко, А.Э. Гумметов. ФТП, 44 (6), 721 (2010).

[13] И.К. Камилов, А.А. Степуренко, А.Э. Гумметов. ФТП, 45 (4), 456 (2011).

[14] И.К. Камилов, А.А. Степуренко, А.Э. Гумметов. ФТП, 46 (7), 918 (2012).

[15] И.К. Камилов, А.А. Степуренко, А.Э. Гумметов. ФТП, 48 (2), 145 (2014).

[16] I.K. Kamilov, A.A. Stepurenko, A.E. Gummetov. Int. J. Modern Phys. Appl., 1 (4), 169 (2015).

[17] И.К. Камилов, А.А. Степуренко, А.Э. Гумметов. ФТТ, 58 (7), 1296 (2016).

[18] А.К. Звездин, В.В. Осипов. ЖЭТФ, 58 (1), 160 (1970).

[19] Э.И. Рашба. УФН, 84 (4), 557 (1964).

[20] C. Hilsum, A.C. Rose-Jnnes. Semiconducting III-V Compounds. (Pergamon Press, Oxford-London-N.Y.-Paris, 1961).

[21] Л.Е. Голуб. Тез. докл. VII Росс. конф. по физике полупроводников (Москва, 18-23 сентября 2005) с. 35.

Редактор Г.А. Оганесян

\section{Spin-orbital interaction and mobility of carriers in the longitudinal InSb autosolition under magnetic fild}

\section{I.K. Kamilov, A.A. Stepurenko, A.E. Gummetov}

Institute of Physics,

Daghestan Science Center,

Russian Academy of Sciences,

367003 Makhachkala, Russia

Abstract The version on spin-orbital interactions of orbitally moving and spin-oriented electrons in the longitudinal InSb autosolition under a longitudinal magnetic field is proposed and considered. The additional energy gained by spin-oriented electrons owing to this interaction is define. The expression are derived and estimated separately for a change in the mobility at the orbital motion of electron under magnetic field and the additive mobility of spin-oriented electrons. The mutual influence coefficients of chagning electron mobility at the orbital motion and the additive mobility of spin-oriented electrons are calculated. 